\title{
Physiotherapy in Kinsbourne syndrome: a case report
}

\author{
DOI: https://doi.org/10.5114/pq.2018.75997
}

\author{
Paulo Roberto Fonseca Junior,,2, Daiane de Lima Santana', Natália Marques Covolo1, \\ Catharine Ranieri Scaransi ${ }^{1}$, Vanessa Donato do Vale ${ }^{3}$ \\ ${ }^{1}$ Faculty of Medical Science, Santa Casa de São Paulo Hospital, São Paulo, Brazil \\ ${ }^{2}$ Nove de Julho University, São Paulo, Brazil \\ ${ }^{3}$ Adult Nursing Sector, Santa Casa de São Paulo Hospital, São Paulo, Brazil
}

\section{Abstract}

Purpose. To evaluate the effect of physiotherapy on ataxia and functional capacity in an individual with Kinsbourne syndrome. Methods. A case study of a 25-year-old male diagnosed with Kinsbourne syndrome undergoing a physiotherapy program in an in-hospital environment for 4 weeks, 5 times a week, with 40-minute sessions.

Results. The results in the Scale for the assessment and rating of ataxia (SARA) score decreased significantly after the physiotherapeutic intervention, revealing a significant reduction of ataxia symptoms. The Functional Independence Measure (FIM) scores differed between the pre- and post-physiotherapeutic intervention, with improvement in the activities of the self-care domain and transfers.

Conclusions. The study showed that after physiotherapy, the patient with Kinsbourne syndrome improved his functional capacity and ataxia pattern, suggesting that a physiotherapy intervention may improve coordination and activities of daily living in people with this rare disease.

Key words: opsoclonus-myoclonus syndrome, cerebellar ataxia, rehabilitation

\section{Introduction}

Kinsbourne syndrome, also known as opsoclonus-myoclonus-cerebellar ataxia syndrome, described in 1962 by Marcel Kinsbourne, is a rare autoimmune neurological disease that begins in childhood (usually at the age of 18 months) or in adult life $[1,2]$. The disease is characterized by acute or subacute onset of axial and appendicular cerebellar ataxia, rapid and multidirectional eye movements (opsoclonus), myoclonus jerks, irritability, sleep disturbance, and other behavioural changes [3, 4]. The myoclonus can affect the trunk, limbs (entirely or partially), or facial muscles [1, 2].

Etiologically, there have been identified idiopathic cases, as well as those associated with viral infections, autoimmune diseases, and, in $50 \%$ of patients, occult neuroblastoma [4]. Some infectious agents involved in this syndrome are Epstein-Barr virus (EBV), enteroviruses (e.g. poliovirus, coxsackievirus), mumps virus, Salmonella, Mycobacterium tuberculosis, Rickettsia, and Plasmodium. Neuroblastoma is the tumour most commonly associated with Kinsbourne syndrome in children; Kinsbourne syndrome may be present in $2-7 \%$ of neuroblastoma cases $[2,5]$.

The mainstay of treatment has traditionally consisted in corticosteroids. A variety of other immunosuppressive treatments have been used, typically in combination with corticosteroids, such as intravenous immunoglobulin (IVIG), cyclophosphamide, cyclosporine, azathioprine, or plasmapheresis $[4,6]$. While the symptoms of the disease usually improve with treatment, a relapsing course is common and long-term neurological sequelae are reported in up to $80 \%$ of patients. This includes impairment in motor, cognitive, language, and/ or behavioural function [3].
As no pharmacologic treatment is available for ataxia dysfunctions, physiotherapeutic intervention currently presents the only therapy to improve them [7]. The benefit of physiotherapeutic intervention is under dispute for patients with degenerative ataxia since the cerebellum is known to play an important role in the generation and adaptation of appropriate patterns of limb movements and dynamic control of balance [8-10]. In a systematic review by Milne et al. [11], evaluating rehabilitation interventions for individuals with genetic degenerative ataxia, 5 randomized controlled trials with 292 participants were included. The rehabilitation interventions involved coordination and balance training, multifaceted inpatient rehabilitation, a cycling regime, balance exercises with technology-assisted biofeedback, respiratory muscle training, and treadmill training. The authors concluded that there was consistent evidence that rehabilitation improved function, mobility, ataxia, and balance in genetic degenerative ataxia.

Recent clinical trials performed with ataxic individuals indicated a significant improvement in functional capabilities, such as self-care or several aspects of gait (like velocity, lateral sway, and intra-limb coordination), and in ataxia symptoms (reduced body sway while sitting and improved posture control mechanisms) after rehabilitation programs [7, 12-14].

However, because Kinsbourne syndrome is a rare disease, implementing rehabilitation is limited owing to lack of evidence supporting its efficacy. In order to help further clarify the physiotherapeutic treatment approach to subjects with Kinsborune syndrome, this study evaluates the response to physiotherapy in a patient diagnosed with Kinsbourne syndrome.

Correspondence address: Paulo Roberto Fonseca Junior, Rua Padre João Gonçalves, 116 - Pinheiros - São Paulo / SP - Brazil - Cep: 05432-040, e-mail: paulofonseca28@gmail.com 


\section{Subjects and methods}

This single-blind case report refers to a young male, aged 25 years, diagnosed with Kinsbourne syndrome. The physiotherapeutic diagnosis was neuromuscular changes leading to axial cerebellar ataxia (cervical and scapular girdle), incoordination of upper and lower limbs (dysdiadochokinesia and dysmetria), and dependence in activities of daily life (ADL). The patient underwent physiotherapy in an in-hospital environment for 4 weeks, 5 times a week. Each session lasted 40 minutes and the treatment applied was based on coordination training, functional activities, and muscle strengthening.

The main strategy of the physiotherapeutic intervention was to activate the mechanisms of balance control and multijoint coordination. Furthermore, the intervention trained the patient ability to select and use visual, somatosensory, and vestibular inputs to preserve and retrain the capability for reacting to unforeseen situations and for avoiding falls as efficiently as possible. The exercises included the following categories: (1) static balance, e.g. standing on 1 leg; (2) dynamic balance, e.g. sidesteps, climbing stairs; (3) whole-body movements to train trunk-limb coordination; (4) movements to train upper and lower limb coordination, physical therapist-facilitated movements of the upper and lower limb; (5) manual role activities that required coordination with postural control; these involved facilitated or independent performance of scapular movements, as well as rotational control; (6) strengthening, performed in the standing, sitting, or lying position and focused on the upper limb, lower limb, or trunk muscles; rubber resistant bands and anti-gravity strengthening, such as calf raises and facilitated movement, were options; the patient performed 2-3 sets of 8-10 repetitions, with the intensity level suitable to ensure correct movement patterns and appropriate eccentric control.

The assessment was performed by an evaluator blinded for the purpose of the study, with the Scale for the assessment and rating of ataxia (SARA) and Functional Independence Measure (FIM). The SARA scale is approved as a valid measure of disease severity and used in degenerative cerebellar and spinocerebellar diseases, as well as in idiopathic ataxias [13-16]. The score includes 8 items: 3 items rating gait and posture, 1 item for speech disturbances, and 4 items for limb kinetic functions. The total score of the scale is 40 points; the higher the score, the worse the performance (see appendix). FIM is an instrument that evaluates the inability of patients with functional restrictions of various causes. It quantitatively assesses the care demand of a person for performing motor and cognitive ADLs, and contains the total of 18 items. Higher FIM scores indicate patients who have a higher level of independence and require less assistance. The 18 items constitute the total score, which ranges from 18 to $126[17,18]$.

\section{Ethical approval}

The research related to human use has been complied with all the relevant national regulations and institutional policies, has followed the tenets of the Declaration of Helsinki, and has been approved by the authors' institutional review board or an equivalent committee.

\section{Informed consent}

Informed consent has been obtained from the individual included in this study.

\section{Results}

The SARA score decreased significantly ( $27 \%$ in total) after the physiotherapeutic intervention, revealing a significant reduction of ataxia symptoms. FIM scores differed between the pre- and post-physiotherapeutic intervention measurements by $16.6 \%$. The activities of the self-care domain, which depend more on the use of arms, were considerably improved, as were transfers, depending on the use of both arms and legs. The low scores for gait and stairs reflect difficulties bound not only with incoordination and ataxia but also with muscle strength and postural balance. The changes in the individual FIM items and SARA scores are reflected in Table 1 and Figures 1 and 2.

\section{Discussion}

In this study, we investigated the related changes in the severity of cerebellar ataxia and disability in ADLs. The combination of the motor manifestations observed in Kinsbourne

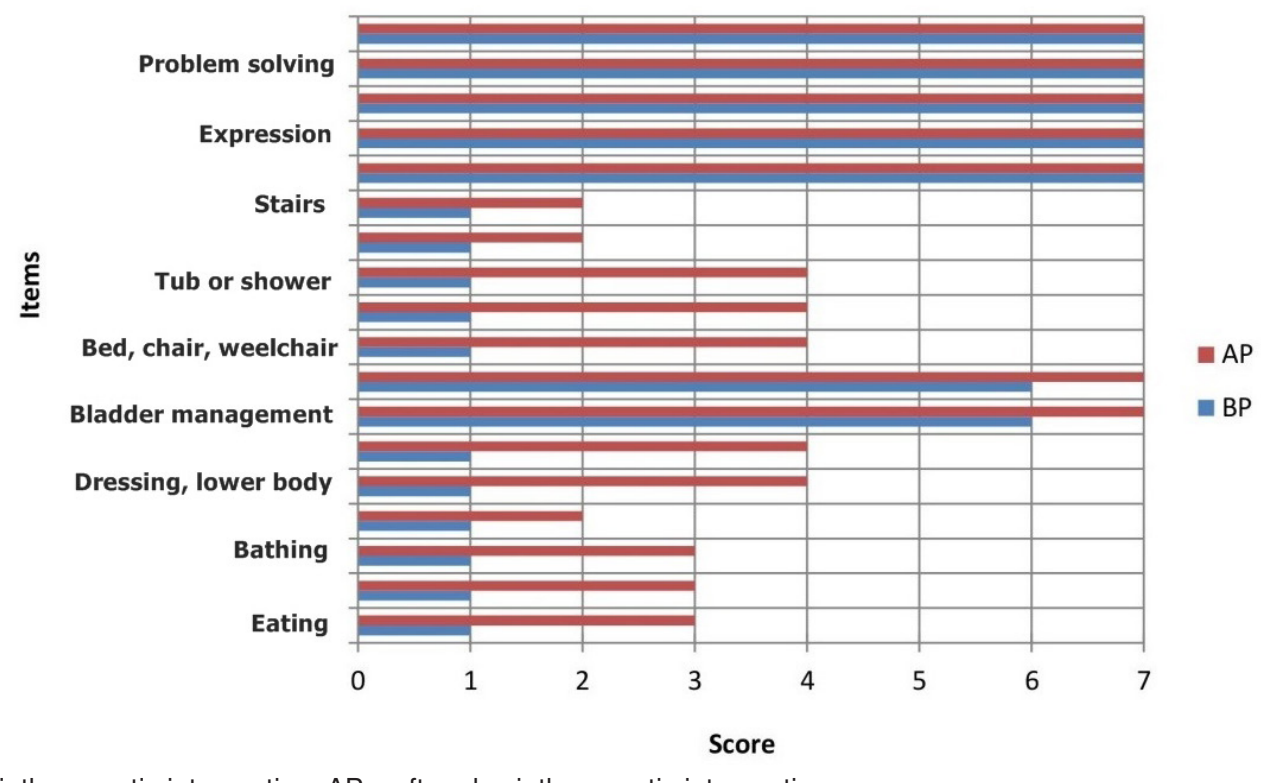

BP - before physiotherapeutic intervention, AP - after physiotherapeutic intervention

Figure 1. Values of the scores in the Scale for the assessment and rating of ataxia before and after physiotherapeutic intervention 


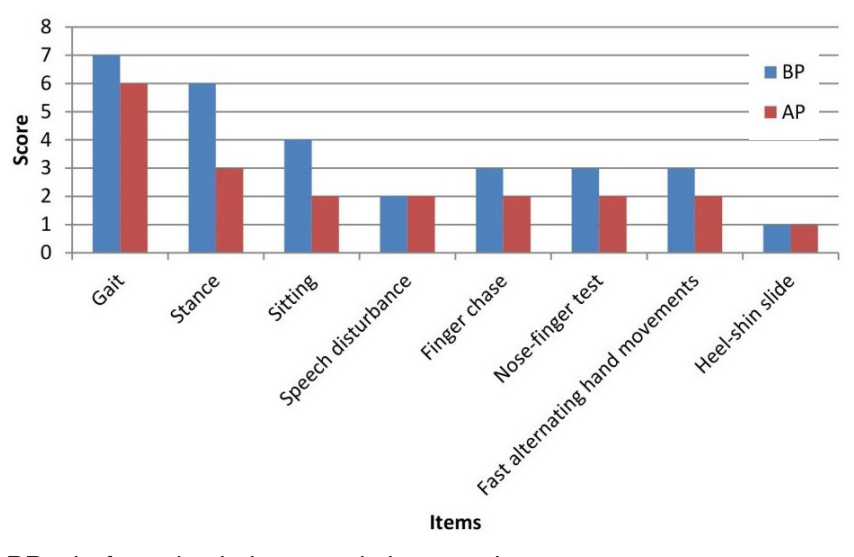

BP - before physiotherapeutic intervention

AP - after physiotherapeutic intervention

Figure 2. Values of the Functional Independence Measure scores before and after physiotherapeutic intervention

syndrome results in the individual's physical incapacity for ambulation or sitting, accompanied by irritability and loss of acquired skills [3].

The rehabilitation program in the described case comprised physical therapy aimed at improving balance, multijoint coordination, and postural control, as well as preventing falls. The patient was also motivated to employ his ability to select and use visual, somatosensory, and vestibular inputs. Compared with the scores before the physiotherapy program, the ones following intervention turned out indicative of clinical improvement. Although the SARA scale does not establish the cut-off value for clinically important changes, the results of the study reveal a significant improvement in motor performance and reduction of ataxia symptoms measured by the SARA clinical scale after the rehabilitation program. The training focused specifically on coordination, increasing motor performance, and reducing ataxia symptoms, enabling the patient to achieve personally meaningful goals in everyday life.

The activities in the mobility subscale of FIM, dependent on the use of legs, were most severely affected. However, those of the self-care domain, which depend more on the use of upper limbs, were considerably improved, as were transfers, depending on the use of both arms and legs.

The rehabilitation program involved strengthening, and the duration of 4 weeks promoted benefits that helped the patient improve some functional aspects, such as gait, static posture control, and sitting. In addition, the intensity of the strengthening exercises, which was reduced to ensure a correct movement technique, may have been favourable for those gains.

\section{Limitations}

There are some limitations of this study, making it difficult to draw definite conclusions. The first limitation relates to the study design: we focused only on one patient, who received physiotherapy in hospital, which restricts generalization. Secondly, one has to remember that patients with more severe impairments are usually not able to perform most of the exercises. Thus, further studies are needed to examine whether patients with more severe impairments, in hospital or in an outpatient setting, would also benefit from a physiotherapeutic intervention (adjusted to their dysfunction, e.g. focused on arm movements) or whether the capacity to improve motor performance relies on a specific level of re-
Table 1. Comparison of the total scores of the SARA and FIM scales before and after physiotherapeutic intervention

\begin{tabular}{|c|c|c|}
\cline { 2 - 3 } \multicolumn{1}{c|}{} & $\begin{array}{c}\text { Before } \\
\text { physiotherapy }\end{array}$ & $\begin{array}{c}\text { After } \\
\text { physiotherapy }\end{array}$ \\
\hline Total SARA score & 31 & 20 \\
\hline Total FIM score & 48 & 69 \\
\hline
\end{tabular}

SARA - Scale for the assessment and rating of ataxia FIM - Functional Independence Measure

paper was not followed up after the 4-week physiotherapy program, so it is not known if the effects obtained in the study persisted in the long-term observation.

\section{Conclusions}

The findings of the study provide evidence to suggest that rehabilitation can improve the health, well-being, and motor function in individuals with Kinsbourne syndrome.

\section{Disclosure statement}

No author has any financial interest or received any financial benefit from this research.

\section{Conflict of interest}

The authors state no conflict of interest.

\section{References}

1. Maranhão MVM, de Holanda ACF, Tavares FL. Kinsbourne syndrome: case report. Rev Bras Anestesiol. 2013;63(3):287-289; doi: 10.1590/S0034-70942013000 300010.

2. Ramos S, Temudo T. Opsoclonus myoclonus syndrome: how long are we going to go on researching? [in Spanish]. Rev Neurol. 2002;35(4):322-325.

3. Galstyan A, Wilbur C, Selby K, Hukin J. Opsoclonus-myoclonus syndrome: a new era of improved prognosis? Pediatr Neurol. 2017;72:65-69; doi: 10.1016/j.pediatrneurol.2017.03.011.

4. Andrade OVB, Troster EJ, Cypel S. Kinsbourne syndrome manifesting with signs of post-viral encephalitis [in Portuguese]. Rev Paul Pediatr. 2011;29(2):300-304; doi: 10.1590/S0103-05822011000200024.

5. Pranzatelli MR. The immunopharmacology of the opsoclonus-myoclonus syndrome. Clin Neuropharmacol. 1996; 19(1):1-47; doi: 10.1097/00002826-199619010-00001.

6. Blaes F, Dharmalingam B. Childhood opsoclonus-myoclonus syndrome: diagnosis and treatment. Expert Rev Neurother. 2016;16(6):641-648; doi: 10.1080/14737175. 2016.1176914.

7. Ilg W, Brötz D, Burkard S, Giese MA, Schöls L, Synofzik M. Long-term effects of coordinative training in degenerative cerebellar disease. Mov Disord. 2010;25(13): 2239-2246; doi: 10.1002/mds.23222.

8. Morton SM, Bastian AJ. Cerebellar control of balance and locomotion. Neuroscientist. 2004;10(3):247-259; doi: $10.1177 / 1073858404263517$.

9. Thach WT, Bastian AJ. Role of the cerebellum in the control and adaptation of gait in health and disease. Prog Brain Res. 2004;143:353-366; doi: 10.1016/S00796123(03)43034-3.

10. Thach WT, Goodkin HP, Keating JG. The cerebellum and the adaptive coordination of movement. Annu Rev Neurosci. 1992;15:403-442; doi: 10.1146/annurev.ne. 15.030192.002155. 
11. Milne SC, Corben LA, Georgiou-Karistianis N, Delatycki MB, Yiu EM. Rehabilitation for individuals with genetic degenerative ataxia: a systematic review. Neurorehabil Neural Repair. 2017;31(7):609-622; doi: 10.1177/ 1545968317712469.

12. Milne SC, Corben LA, Roberts M, Murphy A, Tai G, Georgiou-Karistianis N, et al. Can rehabilitation improve the health and well-being in Friedreich's ataxia: a randomized controlled trial? Clin Rehabil. 2018;32(5):630 643; doi: 10.1177/0269215517736903.

13. Ilg W, Synofzik M, Brötz D, Burkard S, Giese MA, Schöls L. Intensive coordinative training improves motor performance in degenerative cerebellar disease. Neurology. 2009;73(22):1823-1830; doi: 10.1212/WNL.0b013e 3181c33adf.

14. Schatton C, Synofzik M, Fleszar Z, Giese MA, Schöls L, Ilg W. Individualized exergame training improves postural control in advanced degenerative spinocerebellar ataxia: a rater-blinded, intra-individually controlled trial. Parkinsonism Relat Disord. 2017;39:80-84; doi: 10.1016/j.parkreldis.2017.03.016.

15. Weyer A, Abele M, Schmitz-Hübsch T, Schoch B, Frings $M$, Timmann D, et al. Reliability and validity of the scale for the assessment and rating of ataxia: a study in 64 ataxia patients. Mov Disord. 2007;22(11):16331637; doi: 10.1002/mds.21544.

16. Braga-Neto P, Godeiro-Junior C, Dutra LA, Pedroso JL, Barsottini OG. Translation and validation into Brazilian version of the Scale of the Assessment and Rating of Ataxia (SARA). Arq Neuropsiquiatr. 2010;68(2):228230; doi: 10.1590/S0004-282X2010000200014.

17. Aydin T, Taspinar O, Kepekci M, Keskin Y, Erten B, Gunel $M$, et al. Functional independence measure scores of patients with hemiplegia followed up at home and in university hospitals. J Phys Ther Sci. 2016;28(2):553557; doi: 10.1589/jpts.28.553.

18. Prasad R, Hellawell DJ, Pentland B. Usefulness of the Functional Independence Measure (FIM), its subscales and individual items as outcome measures in Guillain Barré syndrome. Int J Rehabil Res. 2001;24(1):59-64; doi: 10.1097/00004356-200103000-00008. 


\section{Appendix. Scale for the assessment and rating of ataxia}

Rater: date:

patient:

\section{Scale for the assessment and rating of ataxia (SARA)}

\section{1) Gait}

a wall including a half-turn (turn around to face the opposite direction of gait) and (2) to walk in tandem (heels to toes) without support.

0 Normal, no difficulties in walking, turning and walking tandem (up to one misstep allowed)

1 Slight difficulties, only visible when walking 10 consecutive steps in tandem

2 Clearly abnormal, tandem walking $>10$ steps not possible

3 Considerable staggering, difficulties in half-turn, but without support

4 Marked staggering, intermittent support of the wall required

5 Severe staggering, permanent support of one stick or light support by one arm required

6 Walking $>10 \mathrm{~m}$ only with strong support (two special sticks or stroller or accompanying person)

7 Walking $<10 \mathrm{~m}$ only with strong support (two special sticks or stroller or accompanying person)

8 Unable to walk, even supported

\section{Score}

\section{Score} feet together in parallel (big toes touching each other) and (3) in tandem (both feet on one line, no space between heel and toe). Proband does not wear shoes, eyes are open. For each condition, three trials are allowed. Best trial is rated.

0 Normal, able to stand in tandem for $>10 \mathrm{~s}$

1 Able to stand with feet together without sway, but not in tandem for $>10 \mathrm{~s}$

2 Able to stand with feet together for $>10 \mathrm{~s}$, but only with sway

3 Able to stand for $>\mathbf{1 0} \mathrm{s}$ without support in natural position, but not with feet together

4 Able to stand for $>\mathbf{1 0} \mathrm{s}$ in natural position only with intermittent support

5 Able to stand $>10 \mathrm{~s}$ in natural position only with constant support of one arm

6 Unable to stand for $>10$ s even with constant support of one arm

\section{3) Sitting}

Proband is asked to sit on an examination bed without support of feet, eyes open and arms outstretched to the front.

0 Normal, no difficulties sitting $>10$ sec

1 Slight difficulties, intermittent sway

2 Constant sway, but able to sit $>10 \mathrm{~s}$ without support

3 Able to sit for $>10 \mathrm{~s}$ only with intermittent support

4 Unable to sit for $>10 \mathrm{~s}$ without continuous support

\section{4) Speech disturbance}

Speech is assessed during normal conversation.

0 Normal

1 Suggestion of speech disturbance

2 Impaired speech, but easy to understand

3 Occasional words difficult to understand

4 Many words difficult to understand

5 Only single words understandable

6 Speech unintelligible / anarthria 
patient:

\section{5) Finger chase}

\section{Rated separately for each side}

Proband sits comfortably. If necessary, support of feet and trunk is allowed. Examiner sits in front of proband and performs 5 consecutive sudden and fast pointing movements in unpredictable directions in a frontal plane, at about $50 \%$ of proband's reach. Movements have an amplitude of $30 \mathrm{~cm}$ and a frequency of 1 movement every $2 \mathrm{~s}$. Proband is asked to follow the movements with his index finger, as fast and precisely as possible. Average performance of last 3 movements is rated.

\section{No dysmetria}

1 Dysmetria, under/ overshooting target $<5 \mathrm{~cm}$

2 Dysmetria, under/ overshooting target $<15 \mathrm{~cm}$

3 Dysmetria, under/ overshooting target $>15 \mathrm{~cm}$

4 Unable to perform 5 pointing movements

\section{6) Nose-finger test}

Rated separately for each side

Proband sits comfortably. If necessary, support of feet and trunk is allowed. Proband is asked to point repeatedly with his index finger from his nose to examiner's finger , which is in front of the proband at about $90 \%$ of proband's reach. Movements are performed at moderate speed. Average performance of movements is rated according to the amplitude of the kinetic tremor.

0 No tremor

1 Tremor with an amplitude $<2 \mathrm{~cm}$

2 Tremor with an amplitude $<5 \mathrm{~cm}$

3 Tremor with an amplitude $>5 \mathrm{~cm}$

4 Unable to perform 5 pointing movements

\begin{tabular}{|l|l|l|l|l|l|}
\hline Score & Right & Left & Score & Right & Left \\
\hline mean of both sides $(R+L) / 2$ & & mean of both sides $(R+L) / 2$ & \\
\hline
\end{tabular}

\section{7) Fast alternating hand movements}

Rated separately for each side

Proband sits comfortably. If necessary, support of feet and trunk is allowed. Proband is asked to perform 10 cycles of repetitive alternation of pro- and supinations of the hand on his/her thigh as fast and as precise as possible. Movement is demonstrated by examiner at a speed of approx. 10 cycles within $7 \mathrm{~s}$. Exact times for movement execution have to be taken.

0 Normal, no irregularities (performs $<10 \mathrm{~s}$ )

1 Slightly irregular (performs $<10$ s)

2 Clearly irregular, single movements difficult to distinguish or relevant interruptions, but performs $<10$ s

3 Very irregular, single movements difficult to distinguish or relevant interruptions, performs $>10 \mathrm{~s}$

4 Unable to complete 10 cycles

\section{8) Heel-shin slide}

Rated separately for each side

Proband lies on examination bed, without sight of his legs. Proband is asked to lift one leg, point with the heel to the opposite knee, slide down along the shin to the ankle, and lay the leg back on the examination bed. The task is performed 3 times. Slide-down movements should be performed within $1 \mathrm{~s}$. If proband slides down without contact to shin in all three trials, rate 4.

0 Normal

1 Slightly abnormal, contact to shin maintained

2 Clearly abnormal, goes off shin up to 3 times during 3 cycles

3 Severely abnormal, goes off shin 4 or more times during 3 cycles

4 Unable to perform the task

\begin{tabular}{|l|l|l|l|l|}
\hline Score & Right & Left & Score & Right \\
\hline mean of both sides $(\mathrm{R}+\mathrm{L}) / 2$ & & mean of both sides $(\mathrm{R}+\mathrm{L}) / 2$ & \\
\hline
\end{tabular}

